This document is the Accepted Manuscript version of a Published Work that appeared in final form in The Journal of Chemical Physics 151, 204304 (2019), copyright @American Institute of Physics after peer review and technical editing by the publisher. To access the final edited and published work see

https://aip.scitation.org/doi/10.1063/1.5123790 


\title{
Excess electron solvation in ammonia clusters
}

\author{
Bence Baranyi and László Turi ${ }^{1}$ \\ Eötvös Loránd University, Institute of Chemistry, Budapest 112, P. O. Box 32, H-
} 1518, Hungary

We performed a combination of quantum chemical calculations and molecular dynamics simulations to assess the stability of various size $\left(\mathrm{NH}_{3}\right)_{n}^{-}$ammonia cluster anions up to $n=32$ monomers. In the $n=3-8$ size range, cluster anions are optimized and the vertical detachment energy of the excess electron (VDE) from increasing size clusters are computed using various level methods including density functional theory (DFT), MP2 and $\operatorname{CCSD(T)}$ calculations. These clusters bind the electron in non-branched hydrogen bonding chains in dipole bound states. The VDE increases with size from a few meV up to $\sim 200 \mathrm{meV}$. The electron binding energy is weaker than in water clusters but comparable to small methanol cluster VDEs. We located the first branched hydrogen bonding cluster that binds the excess electron at $n=7$. For larger $(n=8-32)$ clusters we generated cold, neutral clusters by semiempirical and ab initio molecular dynamics (AIMD) simulations, and added an extra electron to selected neutral configurations. VDE calculations on the adiabatic and the relaxed anionic structures suggest that the $n=12$ - 32 neutral clusters weakly bind the excess electron. Electron binding energies for these clusters $(\sim 100 \mathrm{meV})$ appear to be significantly weaker than extrapolated from

\footnotetext{
${ }^{1}$ E-mail: turilaszlo@ caesar.elte.hu, fax: (36)-1-372-2592.
} 
experimental data. The observed excess electron states are diffuse and localized outside the molecular frame (surface states) with minor $(\sim 1 \%)$ penetration to the nitrogen frontier orbitals. Stable minima with excess electron states surrounded by solvent molecules (cavity states) were not found in this size regime. 


\section{Introduction}

The appearance of the deep blue color after dropping a piece of sodium metal in liquid ammonia is a well-known phenomenon and a favorite subject of undergraduate general chemistry laboratories. More than 30 years after the publication of the observation by Weyl, ${ }^{1}$ Kraus proposed that the species responsible for the color was the solvated electron. ${ }^{2}$ Since then solvated electrons have been observed in several other solvents, most importantly in water. ${ }^{3}$ Although the importance of the hydrated electron is paramount, ${ }^{4}$ the ammoniated electron has still remained a traditional prototype of the solvated electron systems.

Experimental ${ }^{5,6,7,8,9}$ and theoretical studies ${ }^{10,11,12}$ have explored a wide spectrum of physical properties of the ammoniated electron both in bulk liquid and clusters. Still, conceptually important structural questions need more elaborate clarification. These questions concern the way the excess electron is attached to various size ensembles of ammonia molecules. While the smallest ammonia clusters can bind the electron only in a dipole bound fashion, in bulk liquid the excess negative charge is stabilized in the interior of the liquid phase. It is unclear, however, how the transition from the dipole bound state to the internal state takes place as the cluster size increases. Equally importantly, there is no consensus on the precise description of the excess electron distribution in the interior of bulk ammonia, and the way how this charge distribution influences the structure of the solvent phase. ${ }^{10,11}$

The cavity model with a localized excess electron distribution in a solvent void surrounded by properly oriented ammonia molecules, has been the canon for the bulk ammoniated electron's structure for decades. This structural model has its origins in the simple one-electron description of an excess electron embedded in a classical environment. ${ }^{13}$ Works along this route include dielectric continuum models ${ }^{14}$ and one-electron path integral simulations. ${ }^{15,16,17,18}$ Extension of the quantum treatment to the electrons of the solvent provides a more realistic description of the ammoniated electron. Static quantum chemistry calculations 
on finite ammonia cluster anions have been performed at the Hartree-Fock (HF) level, ${ }^{19,20}$ using density functional theory, ${ }^{10}$ and employing highly correlated post-HF ab initio methods. ${ }^{11}$ While most of the calculations were carried out on artificial ammonia clusters mimicking cavity arrangements of the solvent molecules, others also characterized dipole bound anions of small cluster size. ${ }^{11}$ Based on DFT calculations on $\left(\mathrm{NH}_{3}\right)_{n}^{-}$anions $(n=2-8)$ Shkrob suggested that the ammoniated electron may be better understood as a solvent stabilized multimer radical anion with a substantial part of the excess electron density localizing in nitrogen frontier orbitals of the molecules that form the solvent cavity. ${ }^{10}$ Sommerfeld reported coupled cluster ab initio calculations on the smallest possible dipole bound anions ( $n=3$ and 4$)$ and a hexamer model in an arbitrary cavity arrangement, and found only a minor excess electron transfer to the $\mathrm{N}$ frontier orbitals. ${ }^{11}$ Based on his findings he questioned the radical anion model. ${ }^{11}$ It is, however, well understood that observations for small finite size solvated electron models are not necessarily generalizable to large clusters, or the bulk.

The only many-electron based study that attempted to simulate the temporal behavior of the ammoniated electron is the DFT based ab initio molecular dynamics study by Chaban and Prezhdo. ${ }^{12}$ They simulated the dynamics of alkali and alkaline earth metals solvated in a bath of 32 ammonia molecules in a periodic simulation cell. To the best of our knowledge, the dynamics of the ammoniated electron clusters (without the interference of counter ions) have not been investigated using many electron models. Such simulations would be necessary to understand the transition of the electron binding patterns from the smallest dipole bound anionic clusters via middle size cluster anions to the infinitely dilute bulk ammoniated electron.

To fill the hiatus between small cluster quantum chemistry calculations and bulk solvated electron simulations we propose the following sequence of investigations. In the present study, we perform comparative quantum chemistry calculations on small ammonia cluster anions beyond those considered by Sommerfeld. ${ }^{11}$ The aim in this work is to examine 
the dipole bound anions of ammonia clusters, and evaluate the evolution of the binding patterns with increasing cluster size. It is also our purpose to test several less rigorous quantum chemistry methods in comparison with high level ab initio quantum chemistry calculations. Optimistically, the most reliable approximate methods can be transferred for the examination of larger ammonia clusters. Next, we extend our investigations to the temporal characterization of the system by performing semiempirical and ab initio molecular dynamics simulations to generate ensembles of larger neutral clusters $(n=8-32)$. Excess electron binding properties of these clusters will be analyzed to test a) whether one can find neutral configurations that are able to bind an excess electron, b) whether these configurations can be relaxed to stable ammoniated electron clusters, and c) whether one can find different electron binding structural patterns in these clusters. The present study can also be considered as the starting point of our more extensive anionic AIMD simulations that are currently in progress and will be the topic of our subsequent publication.

The structure of the paper will be as follows. In Sec. II, we briefly describe the investigated configurations and the main features of the quantum mechanical and molecular dynamics computational techniques that are employed in the present study. The first part of Sec III collects the results of the benchmarking calculations, while the second part reports the results of our initial dynamical simulations in combination with the subsequent quantum mechanical computations. Sec IV provides an analysis of the results followed by a discussion and conclusions.

\section{Methods}

The present paper begins with a set of comparative quantum chemistry calculations on various size ammonia cluster anions. The investigated structures start from the smallest possible cluster that may bind an excess electron, the dimer. Next, we gradually extend the size of the 
cluster anions up to $n=8$. Various hydrogen-bonding conformations, non-branched (linear) and branched chains will be considered. The applied quantum chemistry includes ab initio methods using coupled-cluster $\operatorname{CCSD}(\mathrm{T})^{21}$ and second-order MP2 perturbation theories, ${ }^{22}$ and DFT methods with the BLYP, ${ }^{23,24}$ LC-BLYP, ${ }^{23,24,25}$ B3LYP, ${ }^{24,26,27}$ BHandHLYP, ${ }^{28,29}$ and PBE0, ${ }^{30,31}$ functional models. These functionals have been employed in several ab initio molecular dynamics studies of aqueous and non-aqueous solvated electrons..$^{32,33,34,35,36}$ This fact motivated our choice. Of the employed theoretical methods, while B3LYP and PBE0 have been demonstrated to overestimate excess electron binding, ${ }^{37}$ the BHandHLYP functional predicted acceptable agreement with MP2 numbers. ${ }^{37,38,39}$ As a general observation, it has been shown that increasing the Hartree-Fock exchange contribution improves the performance of the DFT methods for hydrated electron clusters. ${ }^{38,39}$ The higher HF exchange character of the BHandHLYP functional results in better description of the long range interactions in comparison with BLYP, B3LYP and PBE functionals. ${ }^{38}$ Since these conclusions remained valid in a non-aqueous solvent, methanol, ${ }^{40}$ one may anticipate similar tendencies in ammonia, as well. Alternatively, BLYP (and other generalized gradient approximation (GGA) based functionals) can be improved by using the long-range correction (LC) scheme. ${ }^{25}$ Our observations for methanol cluster anions indicate significant improvement of the VDE using the LC-BLYP method relative to the non-corrected BLYP calculations. ${ }^{40}$ For this reason, we also apply the long-range correction (LC) scheme for the BLYP functional in the present work. Nevertheless, we note that the LC-BLYP method is a particular case of the LC- $\mu$ BLYP procedures with a specific choice of the range separation parameter $\left(\mu=0.47\right.$ bohr $\left.^{-1}\right){ }^{25,41}$ Tuning $\mu$ provides a sensible way to improve the capabilities of the LC- $\mu$ BLYP methods and assess the adequate amount of HF exchange for the investigated system. ${ }^{42}$ Previously we optimized $\mu$ for a small methanol tetramer anion according to the prescription of Baer et al., ${ }^{42}$ and found that application of the optimal range separation parameter $\left(0.25 \mathrm{bohr}^{-1}\right)$ resulted in 
less favorable agreement with high level ab initio VDE values than using LC-BLYP. ${ }^{40}$ Here we further illustrate this problem for ammonia cluster anions anticipating similar conclusions. At the outset we also note that most DFT methods do not account for dispersion energy. ${ }^{43}$ Therefore, varying the HF exchange can be reasonably judged only in the context of spanning the available and acceptable (high level ab initio) data.

Application of sufficiently diffuse and large basis sets is central to the proper description of weakly bound anions. Since the purpose of the present work is the characterization of clusters containing dozens of ammonia molecules, the use of more extensive sets applicable for the smallest clusters may not be feasible here. It is also well understood that less expensive basis sets may provide a reliable representation of the electronic wave function in larger solvated electron clusters. ${ }^{38}$ It is for these reasons that in the quantum chemical calculations we apply the inexpensive 6-31(1+3+)G(d) basis set, and the more extended aug $N$-cc-pVDZ $(N=3,4,5)$ sets. In the $6-31(1+3+) G(d)$ basis set $^{38}$ one adds two additional diffuse s functions on the hydrogen atoms to the standard $6-31++\mathrm{G}(\mathrm{d})$ set, ${ }^{44,45}$ while in the $\operatorname{aug} N$-cc-pVDZ $(N=3,4,5)$ basis $\operatorname{sets}^{38}$ the aug-cc-pVDZ set ${ }^{46,47}$ is augmented by $(N-1)$ extra diffuse s functions on the hydrogen atoms and $(\mathrm{N}-1)$ sets of sp diffuse orbitals on the heavy atoms. The performance and limitations of the $6-31(1+3+) \mathrm{G}(\mathrm{d})$ and the aug3-cc-pVDZ sets have been analyzed previously in hydrated electron cluster calculations. ${ }^{38,39}$ Further aspects have also been noted more recently in studies on excess electron solvation in water ${ }^{37}$ and methanol clusters. ${ }^{40}$ In particular, we found that the VDE computed with the $6-31(1+3+) G(d)$ set is usually overestimated (in some cases by $\sim 15 \%$ ) for more strongly bound water cluster anions relative to aug3-cc-pVDZ basis (regardless of the computational method). ${ }^{37}$ For the quantum chemical calculations on small ( $n$ $=2-8$ ) ammonia clusters, we used the Gaussian09 program package. ${ }^{48}$

For clusters with $n \geq 8$ we performed investigations with a combined application of semiempirical molecular dynamics (MD), ab initio molecular dynamics and quantum chemical 
calculations. First, we prepared neutral ammonia clusters with $n=8,12,16,24$ and 32 molecules, ten clusters of each size. The ammonia molecules were placed randomly in a cubic box of $16 \AA$ side length, and contracted using semiempirical $\mathrm{AM} 1^{49}$ based MD simulations at around $0 \mathrm{~K}$. The AM1 MD simulations were performed using the Gaussian09 program package. ${ }^{48}$ During the contraction, we systematically quenched the kinetics energy of the molecules to zero (in every $125 \mathrm{fs}$ during the $\sim 1 \mathrm{~ns}$ contraction dynamics). The quenched $\sim 0 \mathrm{~K}$ clusters were gradually ( $\sim 50 \mathrm{ps)}$ warmed up to $100 \mathrm{~K}$ by the regular application of MaxwellBoltzmann velocity rescaling steps. After reaching the desired $100 \mathrm{~K}$ temperature, we performed an additional 100 ps AM1 based microcanonical equilibration MD simulation for each cluster. The last configurations of these trajectories were used as the starting points of the AIMD simulations of neutral ammonia clusters. We implicitly assume that the system approaches equilibrium in the AM1 simulation part, and the AIMD portion of the trajectories is used to generate the equilibrium configurations for further statistical analysis.

In AIMD, we use DFT for electronic structure calculations. Although AIMD simulations are carried out here to generate neutral ammonia trajectories, our future purpose is to extend our MD simulations to anionic clusters. Reasonably and consistently, one has to choose a method that can be reliably applied later for solvated electron cluster simulations. We have recently employed BHandHLYP successfully in AIMD simulations of methanol cluster anions. ${ }^{36}$ In a preceding benchmarking study we pointed out that due to its Hartee-Fock exchange component the BHandHLYP/6-31(1+3+)G(d) method provides a reasonable estimate of the VDE in small methanol cluster anions $(n=2-8)$, and the BHandHLYP computed VDE values strongly correlate with those of MP2 using the same basis set. ${ }^{40}$ Here, the argument based on the comparison with MP2/6-31(1+3+)G(d) VDE values is sensibly justified by a previously developed strong correlation between the VDE values at MP2/6-31(1+3+)G(d) and eom-EA-CCSD/aug3-cc-pVDZ levels for small water cluster anions. ${ }^{37}$ Due to its reliable 
description of the aqueous and methanolated excess electron systems, we chose the BHandHLYP functional for use in our AIMD studies to generate neutral ammonia cluster trajectories, and for the quantum mechanical calculations in the subsequent analysis of excess electron binding characteristics to these clusters.

The AIMD simulations were performed using the $\mathrm{CP} 2 \mathrm{~K}$ program package. ${ }^{50} \mathrm{We}$ applied the Gaussian and plane wave method (GPW) in combination with the Goedecker-Teter-Hutter pseudopotentials. ${ }^{51,52}$ In GPW one uses a dual basis set representation (contracted Gaussian functions and auxiliary plane waves) of the electron density to efficiently solve the corresponding Kohn-Sham equations. The localized basis set of the simulations is based on the MOLOPT-TZVP basis, which is optimized for molecular systems in both gas and condensed phases. ${ }^{53}$ We augmented the MOLOPT-TZVP basis by the diffuse functions of the $6-31++\mathrm{G}(\mathrm{d})$ basis set and an additional $s$ orbital on the hydrogen atoms with a coefficient that is one-third of the previous diffuse function of the hydrogen atoms. We denote this basis set by aug1MOLOPT-TZVP. The computational burden of the most expensive part of the computations, the evaluation of the HF exchange energy, is reduced by the application of the Auxiliary Density Matrix Methods (ADMM) ${ }^{54}$ with the diffuse aug-FIT3 basis set. For the plane wave basis set we used an energy cutoff of 480 Ry in the simulations. Each system was placed in a cubic simulation cell with a box size of $25 \AA$. For treating long range interactions in clusters we applied the Martyna-Tuckerman Poisson solver. ${ }^{55}$ Molecular dynamics simulations in the canonical (NVT) ensemble were carried out using $1.0 \mathrm{fs}$ timestep at $100 \mathrm{~K}$ temperature. The temperature was maintained by the Canonical Sampling through Velocity Rescaling (CSVR) thermostat ${ }^{56}$ using a time constant of $100 \mathrm{fs}$. We prepared ten AIMD trajectories of 2.5 ps length for each investigated cluster size, and selected four configurations of each trajectory (at $t=0$ ps, 1 ps, 2 ps and 2.5 ps) providing forty configurations for each cluster size. These configurations span an overall 25 ps sample size, and are assumed to represent thermal 
distribution at $100 \mathrm{~K}$. We performed single point quantum chemical calculations on these configurations with and without the excess electron, and computed the VDE of the anions (equivalently the vertical electron affinities (VEA) of the neutral clusters). At the last step of the analysis, we carried out direct geometry optimization on one of the strongest excess electron binding anions for $n=12$ and 16. Since direct geometry optimization proved to be cumbersome for the $n>16$ cluster anions, we optimized the $n=24$ and 32 cluster anions using simulated annealing molecular dynamics. VDE values were also computed for these relaxed structures. The energy calculations were performed using the Gaussian09 program package with the BHandHLYP functional using the atom centered 6-31(1+3+)G(d) and aug3-cc-pVDZ basis sets. The molecular structures and spin densities were analyzed with the GaussView program of the Gaussian09 program package. The energies and geometries of the relevant structures are collected in the Supplementary Material (SM).

\section{Results}

Small ammonia clusters $(n=3-8)$.

It is well established that the smallest ammonia cluster that binds an excess electron is the trimer. ${ }^{11}$ Nevertheless, at the outset we tested a series of DFT methods to see whether they predict excess electron binding to an ammonia monomer or dimer. For the tests we optimized monomer and dimer ammonia anions using various theoretical methods. Table S1 of the Supplementary Material contains the computed VDE values for the monomer and dimer anions. Of the investigated methods PBE and B3LYP calculate stable monomer and dimer anions, while BLYP predicts stable dimer anions with respect to electron detachment. These functionals appear to fail to predict even the simplest qualitative aspect of the problem, namely the minimal size of the ammonia cluster that is able to bind an excess electron. The LC-BLYP and BHandHLYP methods pass this test along with the ab initio MP2 method, as well. 
Turning toward larger clusters, Table I collects the VDE values for the $n=3-8$ ammonia cluster anions computed in the BHandHLYP/6-31(1+3+)G(d) optimized geometries using several methods and two basis sets, 6-3(1+3+)G(d) and aug3-cc-pVDZ. We note that the VDE values are only marginally affected by full optimizations of the structures using the respective method/basis set combinations relative to single-point VDEs computed in the BHandHLYP/6$31(1+3+) \mathrm{G}(\mathrm{d})$ optimized geometries. We find a single minimum for the trimer anion consistent with the findings of Sommerfeld. ${ }^{11}$ It is a hydrogen bonded non-branched (linear) chain containing a hydrogen acceptor and a hydrogen-donor molecules at the two ends of the chain, and an acceptor-donor molecule in the middle. Cyclic trimer structures do not appear to bind the excess electron. The linear trimer binds the excess electron with a VDE between 20-40 meV consistent with the best available theoretical estimate of $26-28 \mathrm{meV} .{ }^{11} \mathrm{We}$ also performed the trimer and tetramer calculations with $\operatorname{CCSD}(\mathrm{T})$ using the $\operatorname{aug} N$-cc-pVDZ $(N=3,4,5)$ basis sets. The trimer VDE values of $21.7 \mathrm{meV}, 30.6 \mathrm{meV}$ and $31.0 \mathrm{meV}$ for $N=3,4$ and 5, respectively, increase to $62.3 \mathrm{meV}, 66.1 \mathrm{meV}$ and $66.1 \mathrm{meV}$ in the tetramer. The tendency indicates reasonably fast convergence, in particular in increasing size clusters that are in the focus of the present paper. This suggests that the application of the aug3-cc-pVDZ basis set is of sufficient precision for our purposes.

The VDE values of Table I indicate increasing excess electron binding as the clusters grow. This effect is clearly demonstrated in Figure 1 showing the aug3-cc-pVDZ VDE values for the $n=3-8$ linear clusters using various methods. Apparently, the $\operatorname{CCSD}(\mathrm{T})$ results are nicely approached by the LC-BLYP and the BHandHLYP values. As a typical example, we report VDE values in the 150-200 meV range for the strongest binding optimized linear octamer chain using BHandHLYP and LC-BLYP. We also tested briefly the performance of the LC$\mu \mathrm{BLYP}$ method with optimized range separation parameters for $n=3-5$ linear cluster anions. The optimization according to Baer et al. ${ }^{42}$ results approximately uniform $\mu$ values for these 
systems ( 0.3), but the calculated VDE values are usually significantly higher (especially for smaller systems and smaller basis sets) than those calculated using LC-BLYP and CCSD(T) (see Table S2, the Supplementary Material). This behavior is consistent with that found previously for a methanol tetramer anion. ${ }^{40}$ Thus, on the one hand, we do not observe significant system, size and basis set dependence for the range separation parameter, but, on the other hand, the optimal $\mu$ shifts the VDE values unfavorably. Since this latter tendency needs further investigations, we will not pursue the use of the LC- $\mu$ BLYP method in the present work. All other investigated DFT methods (PBE, BLYP, B3LYP) fall far off with significant overestimation. Although MP2 does a reasonable job, the two better performing DFT methods seem preferable. We also note that application of the $6-31(1+3+) \mathrm{G}(\mathrm{d})$ results in slightly higher VDE values, and this overestimation should be kept in mind when using this cheaper basis set for more extensive systems. We reached similar conclusions in our quantum chemical and AIMD studies of negatively charged methanol clusters. ${ }^{36,40}$

The most stable linear configurations contain only single hydrogen-bond acceptor/single donor molecules within the chains. Such structures are illustrated in Figures 2 and 3 with their representative spin density isosurfaces. The excess electron localizes in a diffuse dipole bound state at the three dangling hydrogens of the hydrogen-bond acceptor end of the chains. These states are similar to that reported by Sommerfeld for the trimer anion. ${ }^{11}$ Figure 2 shows three isosurfaces that contain $50 \%$ of the total spin for the optimized trimer, pentamer and heptamer structures (with isovalues of $0.000036,0.000042$ and 0.000044 , respectively). As the clusters grow the VDE increases, and the diffuse excess states slightly shrink. Nevertheless, the excess electron distribution remains quite sizeable even in the octamer structure, completely covering the ammonia molecule at the end of the chain, and surrounding at least one other monomer. Figure 3, showing the spin density surfaces in the octamer at selected isovalues, illustrates the degree of localization of the excess electron in and around the cluster. The first isosurface with 
a spin density value of 0.0004 indicates electron localization on the last three ammonia molecules at the acceptor end of the chain. This portion of the spin density appears to overlap with and penetrate to the molecular orbitals (frontier orbitals) on the nitrogen atoms. The enclosed spin density accounts only for $\sim 1 \%$ of the total spin density indicating a (numerically) relatively minor effect. Decreasing the isovalue to 0.0003 , the spin density starts appearing outside the terminal hydrogen atoms and forms parts of the dipole bound surface state of the excess electron. The contribution of this portion gives less than another $1 \%$ of the spin density. Successive decrease of the spin density isovalues to $0.0002,0.0001$, and 0.00005 further contributes to the gradual increase of the diffuse surface part of the electron distribution; the resulting isosurfaces surround $\sim 8 \%, 27 \%$ and $45 \%$ of the total spin density, respectively. The last surface of Figure 3 (see also Figure 2) completely surrounds the terminal ammonia molecule that, nonetheless, is located in a tight excluded cavity for the excess electron within the diffuse excess electron cloud.

In addition to the non-branched hydrogen bonded structures, we located anionic isomers with branched hydrogen bonding network for the $n \geq 7$ cluster sizes. We show two such structures in Figure 4 for $n=7$ and 8, with their spin isodensity surfaces (corresponding to 50\% of the total density). Interestingly, for the $n=7$ case, the branched structure appears to be more stable than the linear one, but its VDE is lower $(75.2 \mathrm{meV}$ vs. $157 \mathrm{meV}$, BHandHLYP with aug3-cc-pVDZ). For the $n=8$ isomer, the branched structure has a higher VDE than its linear counterpart (260 meV vs. $175 \mathrm{meV}$, BHandHLYP with aug3-cc-pVDZ). In both cases, the electron is bound to dangling hydrogens of at least two ammonia molecules. It should be emphasized that we have not explored all possible branched isomer structures, nor, in particular, the factors connecting the relative stability, the hydrogen bonding structure and the excess electron binding strength of the anions. Nevertheless, we believe that these branched clusters can be seen as representing the transition from the linear chain motif to non-linear, branched 
configurations that are likely to become dominant in population (probability) as the clusters grow.

Intermediate size ammonia clusters $(n=8$-32).

To investigate the excess electron binding to larger clusters, we prepared ultracold clusters at $\sim 0 \mathrm{~K}$, increasing in size from $n=8$ to 32 , and gradually heated them up to the temperature of the analysis, $\sim 100 \mathrm{~K}$. We built ten independently prepared configurations of each investigated size. After the cluster preparation procedure we equilibrate the clusters at 100 $\mathrm{K}$, launching consecutive $100 \mathrm{ps}$ AM1 based MD and 2.5 ps AIMD trajectories starting from each prepared cluster configuration. For further calculations and the analysis we select four configurations from each of the ten AIMD trajectories for each cluster size. We find that in these molecular aggregates the molecules pack more tightly than in the elongated chains. Figure 5 shows characteristic examples for the examined cluster configurations. We find that the neutral clusters remain compact and thermally stable at $100 \mathrm{~K}$ with respect to autodissociation. Interestingly, with increasing cluster size ordered motifs start appearing in the clusters, in a quasi-crystalline order. Figure 5 also illustrates such a sample configuration for the $n=32$ size.

We computed the interaction energy between the selected neutral configurations of the AIMD trajectories and one excess electron (VDE of the anion). These calculations represent the simplest possible model of the electron attachment process, where we add zero kinetic energy electrons to a thermal distribution of neutral clusters. Similar scenarios were analyzed for water and methanol clusters previously. ${ }^{40,57,58} \mathrm{We}$ note that the calculations of this section are performed using the BHandHLYP functional with the 6-31(1+3+)G(d) basis set. We already illustrated in the previous section that BHandHLYP/6-31(1+3+)G(d) and BHandHLYP/aug3cc-pVDZ methods/basis sets reliably approximate the energetics of excess electron attachment to small ammonia clusters, although the former one slightly overestimates the VDE. We found 
that for the smallest investigated cluster size $(n=8)$ the VDE values are negative in all sampled cases, suggesting that excess electrons are highly unlikely to bind to the smallest, cold ammonia clusters. The $n=12$ clusters have a definite tendency to attract the electron. Of the forty computed $\left(\mathrm{NH}_{3}\right)_{12}^{-}$cases, $\sim 70 \%$ of the configurations are more stable in the anionic form than in the neutral one. The typical interaction strength varies around $\sim 20 \mathrm{meV}$ for the binding configurations. As the clusters grow, the electron binding tendency further enhances with VDE magnitudes as high as $\sim 80 \mathrm{meV}, \sim 130 \mathrm{meV}, \sim 210 \mathrm{meV}$ for $n=16,24$ and 32, respectively. For the two largest examined cluster sizes ( $n=24$ and 32), practically all considered neutral clusters bind the electron. The electron distribution, however, remains extremely diffuse and delocalized in all cases. As Figure 6 and Figures S1-S3 demonstrate, an extra electron attached to a neutral ammonia cluster essentially engulfs the entire cluster.

As the last step of the analysis we optimized selected anionic ammonia clusters that were prepared initially by adding an electron to a neutral cluster configuration. Note that these configurations very weakly bind the extra electron. Since we experienced severe convergence problems during the optimization of the resulting weakly bound anions, for the optimization we chose one of the least weakly binding neutral geometries for $n=12,16,24$, and a relatively strongly binding, non-crystalline one for the $n=32$ cluster. Direct geometry optimization was successfully performed for the $n=12$ and 16 anions. The electron binding energy significantly grew from the initial $52 \mathrm{meV}$ to $134 \mathrm{meV}$ in the optimized structure for $\left(\mathrm{NH}_{3}\right)_{12}^{-}$. This optimized anion contains a longer hydrogen-bonded chain motif that binds the excess electron relatively strongly, similar to the hydrogen-bonded chains studied above (see Figure S1 in the Supplementary Material). The $n \geq 16$ optimized cluster anions, lacking longer linear hydrogen bonded motifs, remain more compact (Figure 6 and Figures S2 and S3). Although these clusters relax sizably in energy during the optimization, their electron binding energies do not change significantly. This indicates that the relaxation energy of the anionic cluster during the 
optimization is approximately the same as the energy relaxation of the neutral cluster within the anion. The hydrogen-bonding network of the investigated configurations appears to be stiff enough to disallow significant nuclear re-arrangement and coupled excess electronic relaxation. Figure 6 compares the spin density isosurfaces after the initial attachment of an excess electron to a neutral $n=16$ cluster, and after optimization. Geometry relaxation leads the initial cluster with a highly delocalized excess electronic state to a structure that accommodates the electron in a somewhat more localized state. The binding energy of the electron, however, is roughly the same before and after optimization $(\sim 100 \mathrm{meV})$. It is also worth noticing that, although geometry relaxation results in a somewhat more localized excess electron state, in both cases most of the spin density of the excess electron is located outside the molecular frame of the cluster. The excess electron clearly occupies a surface state. We observe similar tendencies for $\left(\mathrm{NH}_{3}\right)_{24}^{-}$and $\left(\mathrm{NH}_{3}\right)_{32}^{-}$clusters, although in these two cases we were unable to perform direct geometry optimization due to convergence problems. We, therefore, minimized the energy of the anions using simulated annealing molecular dynamics by continuously decreasing the nuclear kinetic energy of the cluster during the dynamics. The relaxed clusters are shown in Figures S2 and S3 in the Supplementary Material. It is striking in these two cases that no sign of excess electron localization is observed in the relaxed structures. In fact, the electron binding in the relaxed configurations becomes slightly weaker relative to the binding in the initial structures with VDE values of $77 \mathrm{meV}$ (vs. $107 \mathrm{meV}$ initial VDE) and $80 \mathrm{meV}$ (vs. $104 \mathrm{meV}$ initial VDE) for the $n=24$ and 32 cluster anions, respectively.

\section{Discussion and Conclusions}

We performed a series of comparative VDE calculations to characterize the electron binding properties of finite size ammonia clusters. The analysis started from the smallest size ammonia cluster anion of three monomers. Addition of an increasing number of monomeric 
units leads to a hydrogen-bonded chain of molecules with an extra electron attached to the last hydrogen-bond acceptor unit. The interaction can be characterized as a dipole-bound interaction of the electron with the molecular frame. The vertical detachment energy of the relaxed anions increases as the clusters grow, reaching $200 \mathrm{meV}$ in the octamer chain. We also investigated branched cluster anions with $n=7$ and 8 . The VDE in one of these cases reached as high as $\sim 300 \mathrm{meV}$. For comparison, ammonia cluster anion VDE values are lower than the VDE's for similar size water cluster anions ${ }^{59}$ but similar to those found for small methanol cluster anions. ${ }^{40}$ The excess electron distribution as mapped by the spin density appears to be very diffuse and concentrated on the hydrogen bond acceptor end of the chains that possesses free dangling hydrogen atoms. It is interesting to note that most of the excess electron density is located outside the molecular frame, but it also completely surrounds the last one or two monomeric units of the chain. Furthermore, only $\sim 1 \%$ of the excess density is localized directly on the vicinity of the core orbitals of the nitrogen atoms of these monomers. This estimate is in good agreement with that of Sommerfeld, ${ }^{11}$ who, based on similar data, challenged the multimer radical anion picture of Shkrob. ${ }^{10}$

We also investigated the microscopic details of excess electron binding to cold, neutral clusters. The interaction of an excess electron with a preformed neutral cluster may model the very initial step of the electron attachment to form cluster anions. We notice that while the geometry optimized linear $n=8$ anionic chain has a relatively large VDE, neutral $n=8$ clusters taken from MD simulations do not appear to bind the electron. The two sampling procedures, however, are principally different. Geometry optimization produces anionic configurations at $\sim 0 \mathrm{~K}$, a regime that is practically inaccessible experimentally. MD simulations, however, sample from the actual (neutral) thermal distributions, in this case, at $\sim 100 \mathrm{~K}$, producing mainly unstable structures (with respect to electron attachment). This is the main reason why the excess electron stabilization turns out to be significantly greater in small, optimized $0 \mathrm{~K}$ clusters than 
in finite temperature ensembles of intermediate size neutral molecules. Our most evident example demonstrates that while an optimized, linear chain of the $n=8$ anion binds the electron with $\sim 200 \mathrm{meV}$ VDE, electron attachment to ensembles of 8 neutral molecules at $\sim 100 \mathrm{~K}$ appears to be energetically disfavored. The tendency to bind an excess electron for these latter neutral clusters, however, enhances with increasing cluster size. We found the minimal size of the $\sim 100 \mathrm{~K}$ cold neutral clusters that are able to bind an excess electron to be $n \sim 12$. Interestingly, the strength of the initial interaction is found to be stronger in ammonia clusters than in similar size clusters of methanol, but weaker than in water. This is consistent with the picture that neutral methanol clusters are covered on the surface mainly by the apolar methyl groups. ${ }^{36,40}$ The excess electron in all examined cases localized mainly outside the cluster, in a very diffuse state with predominant surface character. Initial localization in the interior of the ammonia clusters is not observed. Electron attachment is followed by a subsequent nuclear and electronic relaxation. During this process the electron can either autodetach from the cluster leaving a neutral cluster behind, or stabilize in the anionic species with increasing VDE and more localized electron distribution. We found that at the temperature of our simulations, the stiff hydrogen bonding network basically hinders the relaxation of the excess electron. The computed VDE values of the anionic structures are anticipated to be comparable to those inferred from the linear relationship for the $n^{-1 / 3}$ size dependence of the experimental VDE tendency found by Sarkas et al. ${ }^{8}$ Although the smallest experimentally observed ammonia cluster anions are larger $(n=41)$ than computed in the present study, the experimental tendency can be extrapolated to the investigated smaller size regime for comparative purposes. The experimental trend predicts VDE values of $290 \mathrm{meV}, 410 \mathrm{meV}$ and $490 \mathrm{meV}$ for $n=16,24$ and 32 , respectively, greater than the strongest computed initial binding energies of $76 \mathrm{meV}, 128$ $\mathrm{meV}$ and $210 \mathrm{meV}$ for the same size clusters. Geometry optimization and/or simulated annealing do not appear to bring the initial VDE values closer to the experimentally 
extrapolated ones. These tendencies are illustrated in Figure 7. We emphasize that both the initial and the relaxed clusters still bind the electron in states that are mostly localized outside the cluster, in surface states. Experimentally, it has been demonstrated for water and methanol clusters that varying the conditions of the cluster preparation procedure leads to different tendencies of the VDE with cluster size. The different trends strongly suggest the existence of different electron binding motifs. Vice versa, it is generally accepted that different excess electron binding motifs of cluster anions are likely to lead to different tendencies of the VDE with cluster size. The sizeable deviation of our calculated results from experiments strongly indicates that experiments see different binding motifs for the excess electron in ammonia clusters. In fact, Sarkas et al. call these states embryonic ammoniated electrons and observe that the cluster VDE extrapolation to infinite size is close to the photoelectric threshold energy of bulk ammoniated electrons implying excess electron distribution embedded in the interior of the clusters. One possible reason for the detected differences between experiment and theory may originate from temperature effects. It is sensible to assume that higher cluster temperatures may provide sufficient energy for VDE enhancement via relaxation and/or isomerization. Another, equally appealing argument, suggests that the electrons may not be attached directly to preformed clusters, but instead they may interact with smaller clusters and monomers during the progressing cluster formation. The idea of selectivity in electron attachment was recently suggested and successfully tested experimentally for water cluster anions. ${ }^{60}$

At the end of the paper, we underline the limitations of our approach. First, we note the approximations involved in the applied computational methods, and the limited sampling. More importantly, the observed differences between theory and the extrapolated experimental numbers may originate from the simplifications in modeling the electron attachment process, zero kinetic energy electrons attached to thermally distributed clusters. Deviations from the assumed model conditions in real experiments might lead to different outcomes. In a related 
context, geometry optimization starting from other initial configurations (conditions) than examined in the present model may lead to relaxed anionic configurations that bind the electron significantly more strongly. We have not considered configurations in the present work beyond those dictated by our simple electron attachment picture. As an alternative direction, we plan to perform molecular dynamics simulations starting from the initially preformed anions (or other alternative, but physically feasible geometries) and directly follow the relaxation trajectories. Similarly, MD simulations initiated from cluster anions with preformed interior state electrons may represent a model of the final state of an electron attachment that takes place during the formation of the clusters. We plan to pursue these routes in our forthcoming work. It will be clearly a future challenge to see how the VDE tendencies may evolve during the relaxation of these intermediate size clusters, and how these tendencies can be corroborated with available experimental data.

\section{Supplementary Material}

Additional VDE values and spin densities of the structures discussed in the text can be found in the Supplementary Material (Part1, Tables S1-S2 and Figures S1-S3). The energies and geometries of the relevant structures are also collected in the Supplementary Material (Part 2).

\section{Acknowledgments}

This work was supported by research grants to L.T. from the National Research, Development and Innovation Office, Hungary (NKFIH, grant number K128136). The computations were performed on the supercomputers of the National Information Infrastructure Development (NIIF) Institute of Hungary. 
Table I. VDE values of linear ammonia cluster anions with different methods and basis sets.

Calculations were performed at the BHandHLYP/6-31 $(1+3+) \mathrm{G}(\mathrm{d})$ optimized structures (bold). Energies are in meV units.

\begin{tabular}{|c|c|c|c|c|c|c|}
\hline \multirow[t]{2}{*}{ Basis set } & \multicolumn{6}{|c|}{ Cluster size, $n$} \\
\hline & 3 & 4 & 5 & 6 & 7 & 8 \\
\hline \multicolumn{7}{|c|}{ BHandHLYP } \\
\hline $6-31(1+3+) G(d)$ & 32.0 & 78.3 & 118 & 148 & 171 & 190 \\
\hline aug3-cc-pVDZ & 31.4 & 72.2 & 108 & 135 & 157 & 175 \\
\hline aug4-cc-pVDZ & 60.6 & 92.4 & 122 & 144 & 163 & 179 \\
\hline aug5-cc-pVDZ & 83.1 & 114 & 140 & & & \\
\hline \multicolumn{7}{|c|}{ BLYP } \\
\hline $6-31(1+3+) G(d)$ & 121 & 183 & 231 & 275 & 308 & 339 \\
\hline aug3-cc-pVDZ & 150 & 204 & 247 & 287 & 317 & 345 \\
\hline \multicolumn{7}{|c|}{ LC-BLYP } \\
\hline $6-31(1+3+) G(d)$ & 20.8 & 65.3 & 103 & 131 & 154 & 171 \\
\hline aug3-cc-pVDZ & 16.6 & 55.6 & 89.1 & 115 & 137 & 154 \\
\hline \multicolumn{7}{|c|}{ B3LYP } \\
\hline $6-31(1+3+) G(d)$ & 152 & 207 & 252 & 288 & 317 & 340 \\
\hline aug3-cc-pVDZ & 156 & 207 & 247 & 273 & 301 & 324 \\
\hline \multicolumn{7}{|c|}{ PBE } \\
\hline $6-31(1+3+) G(d)$ & 183 & 244 & 296 & 338 & 375 & 408 \\
\hline aug3-cc-pVDZ & 196 & 241 & 301 & 326 & 359 & 393 \\
\hline \multicolumn{7}{|c|}{ MP2 } \\
\hline $6-31(1+3+) G(d)$ & -0.5 & 39.4 & 73.5 & 99.0 & 120 & 137 \\
\hline aug3-cc-pVDZ & 3.2 & 37.7 & 67.8 & 91.4 & 111 & 127 \\
\hline \multicolumn{7}{|c|}{$\operatorname{CCSD}(\mathrm{T})$} \\
\hline aug3-cc-pVDZ & 21.7 & 62.3 & 97.3 & 124 & 147 & 164 \\
\hline aug4-cc-pVDZ & 30.6 & 66.1 & & & & \\
\hline aug5-cc-pVDZ & 31.0 & 66.1 & & & & \\
\hline
\end{tabular}




\section{Figure Captions}

Figure 1. Computed VDE values of linear chains of ammonia cluster anions using the aug3cc-pVDZ basis set. Geometries were optimized at the BHandHLYP/6-31(1+3+)G(d) level (see also Table I).

Figure 2. Mesh representation of the spin density isosurfaces of the optimized ammonia trimer (a), pentamer (b) and heptamer (c) anions containing $50 \%$ of the total spin density with isovalues of $0.000035,0.000042$ and 0.000044 , respectively. Note that the last ammonia monomers of the chains are buried beneath the spin density isosurfaces.

Figure 3. Spin density isosurfaces of the optimized ammonia octamer anion with isovalues of (a) 0.0004 , (b) 0.0003 , (c) 0.0002 , (d) 0.0001 and (e) 0.00005 containing $1.0 \%, 1.3 \%, 7.5 \%$, $26 \%$ and $46 \%$ of the total spin density (from top to bottom). Solid surfaces help to visualize small surrounded volumes.

Figure 4. Mesh representation of the spin density isosurfaces of optimized ammonia (a) heptamer and (c) octamer anions with branched hydrogen-bonded systems. The surfaces contain $50 \%$ of the total spin density (isovalues of (b) 0.000037 and (d) 0.000075 ).

Figure 5. Selected neutral ammonia cluster configurations containing (a) 8, (b) 16 and (c and d) 32 ammonia molecules as generated from AIMD simulations. These structures are used for VDE calculations. Note (c) the disordered and (d) the quasi-crystalline configurations for $n=$ 32.

Figure 6. Mesh representation of the spin density isosurfaces for a typical $n=16$ ammonia cluster (a) after an initial electron attachment to the neutral cluster, and (b) for the same cluster after geometry optimization. The surfaces contain $50 \%$ of the total spin density with isovalues of 0.00002 and 0.000025 .

Figure 7. VDE values for various size ammonia cluster anions. Stars: data points extrapolated from the experimentally determined linear VDE vs. $n^{-1 / 3}$ relationship. Blue squares: average 
computed binding energy (at BHandHLYP/6-31(1+3+)G(d) level) of an electron attached to a sample of neutral configurations taken from AIMD simulations. Red circles: the strongest computed binding energy of an electron attached to a sample of neutral configurations. 
Figure 1.

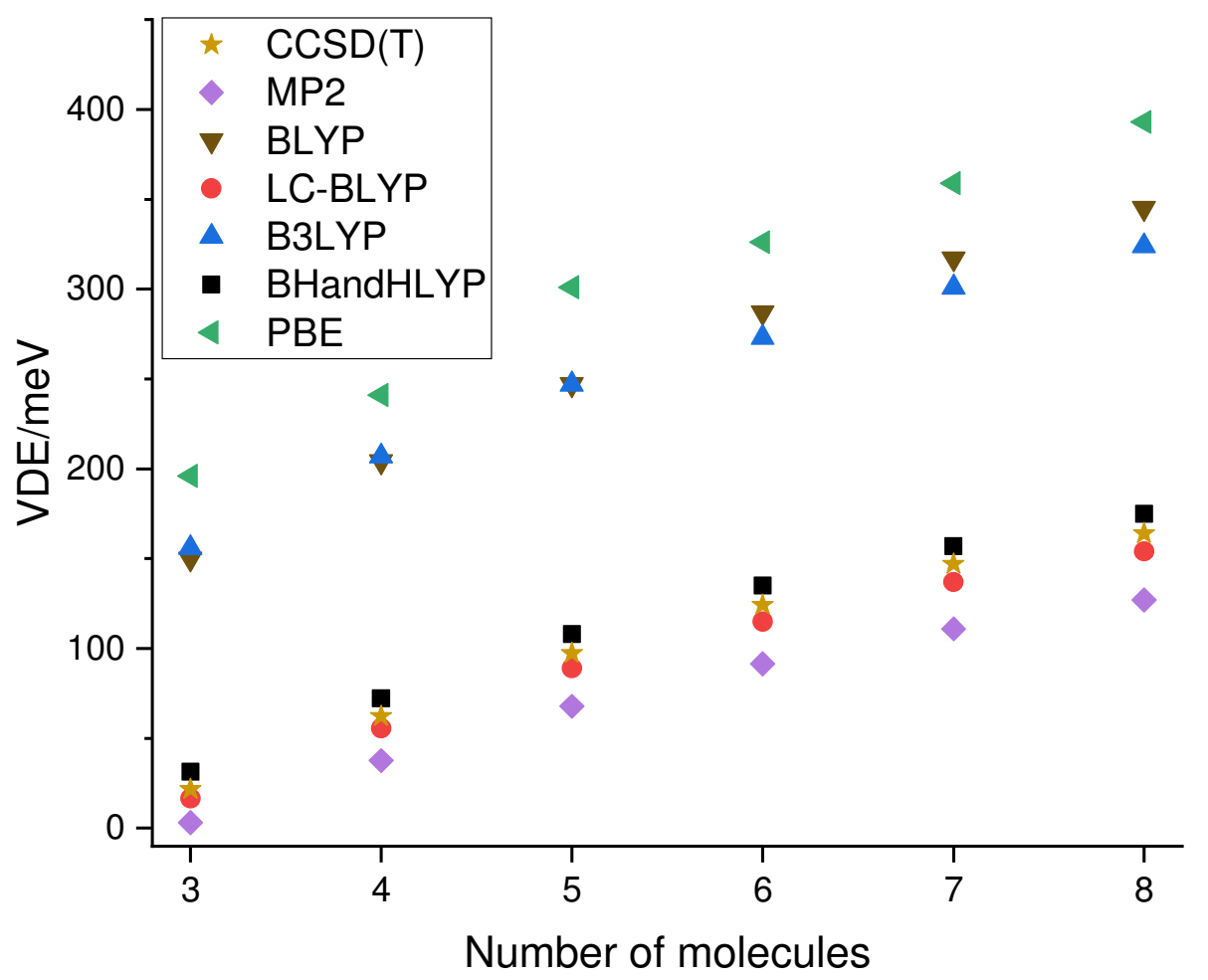


Figure 2.

a)

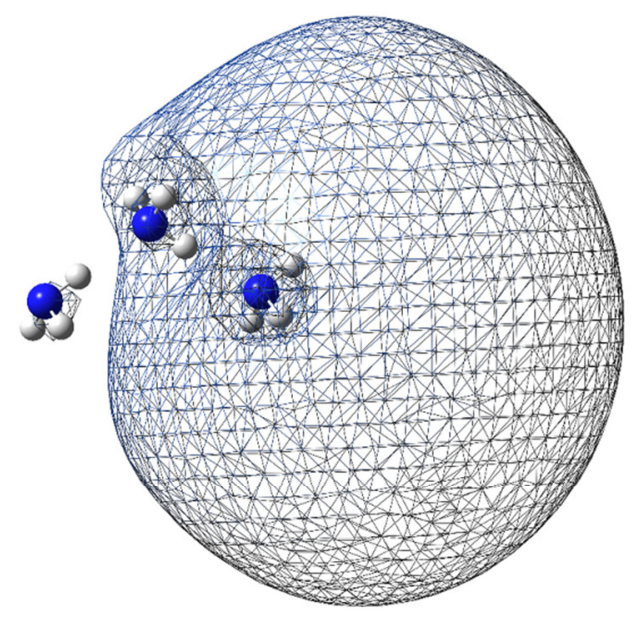

b)

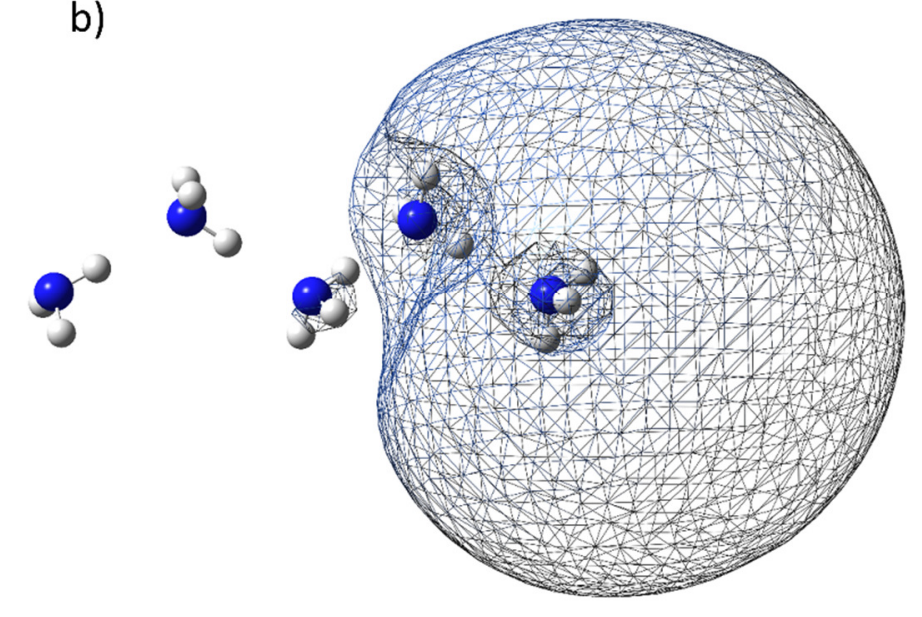

c)

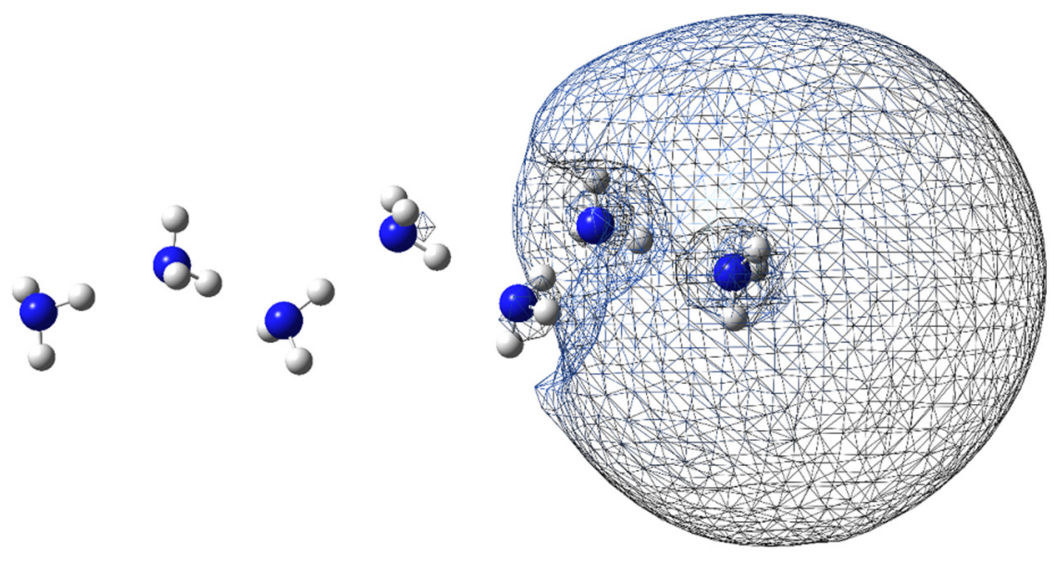


Figure 3.

a) $a^{3}$

b) 9.90

c) $\sigma^{2}, 9,8$

d)

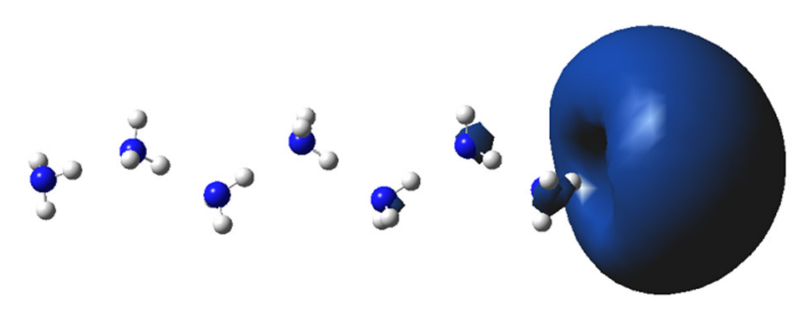

e)

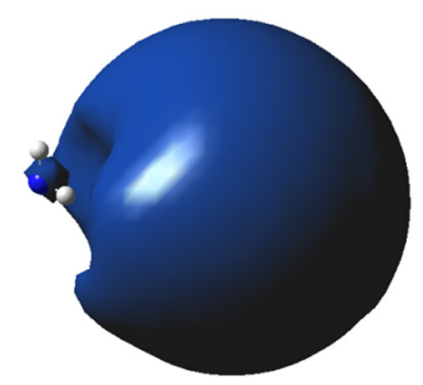


Figure 4.

a)

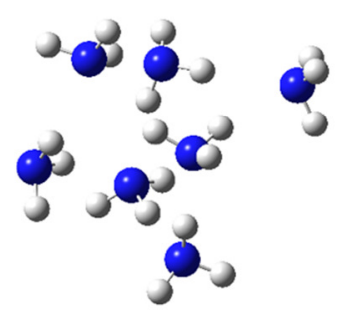

c)

$$
\frac{a^{2}}{a-2}
$$

b)

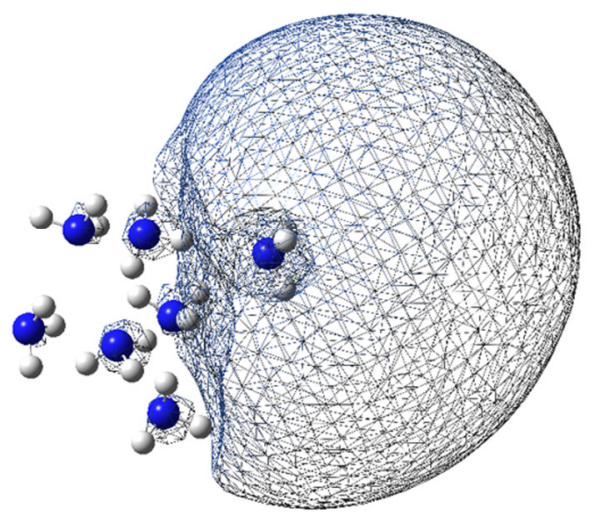

d)

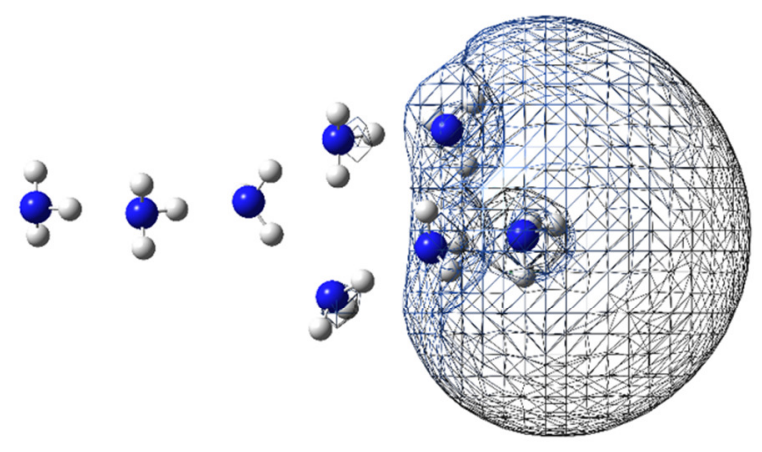


Figure 5.

a)

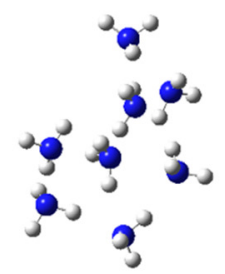

b)

$$
\begin{aligned}
& \text { "ُ } \\
& \text { o }
\end{aligned}
$$

8;

c)

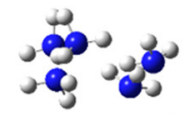

$$
\begin{aligned}
& \text { ودرة }
\end{aligned}
$$

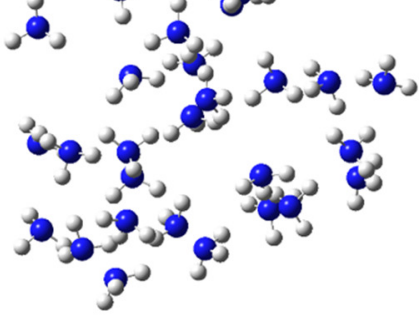

d)

$$
\begin{aligned}
& \text { ¿ : }
\end{aligned}
$$

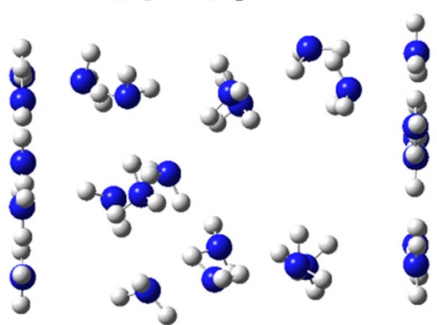

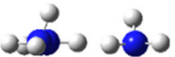


Figure 6.

a)

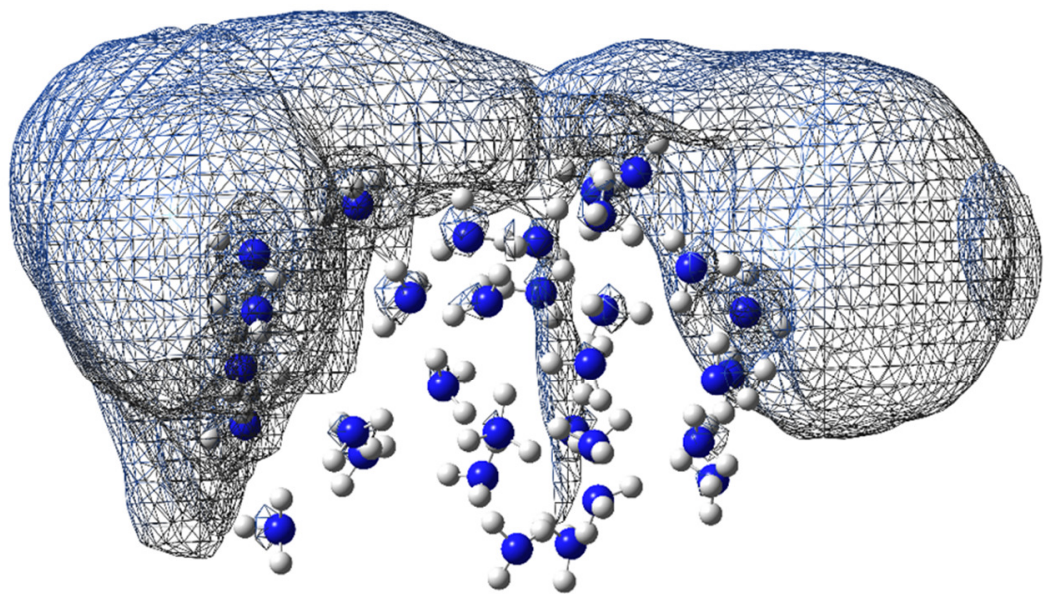

b)

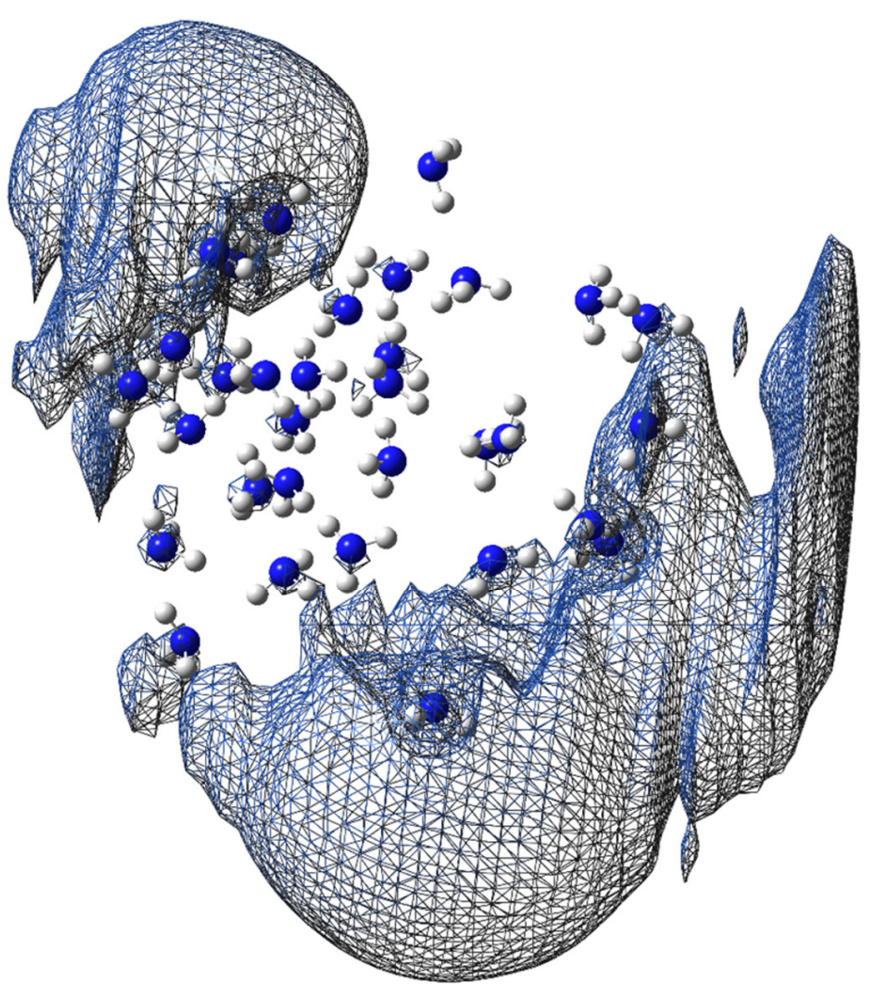


Figure 7.

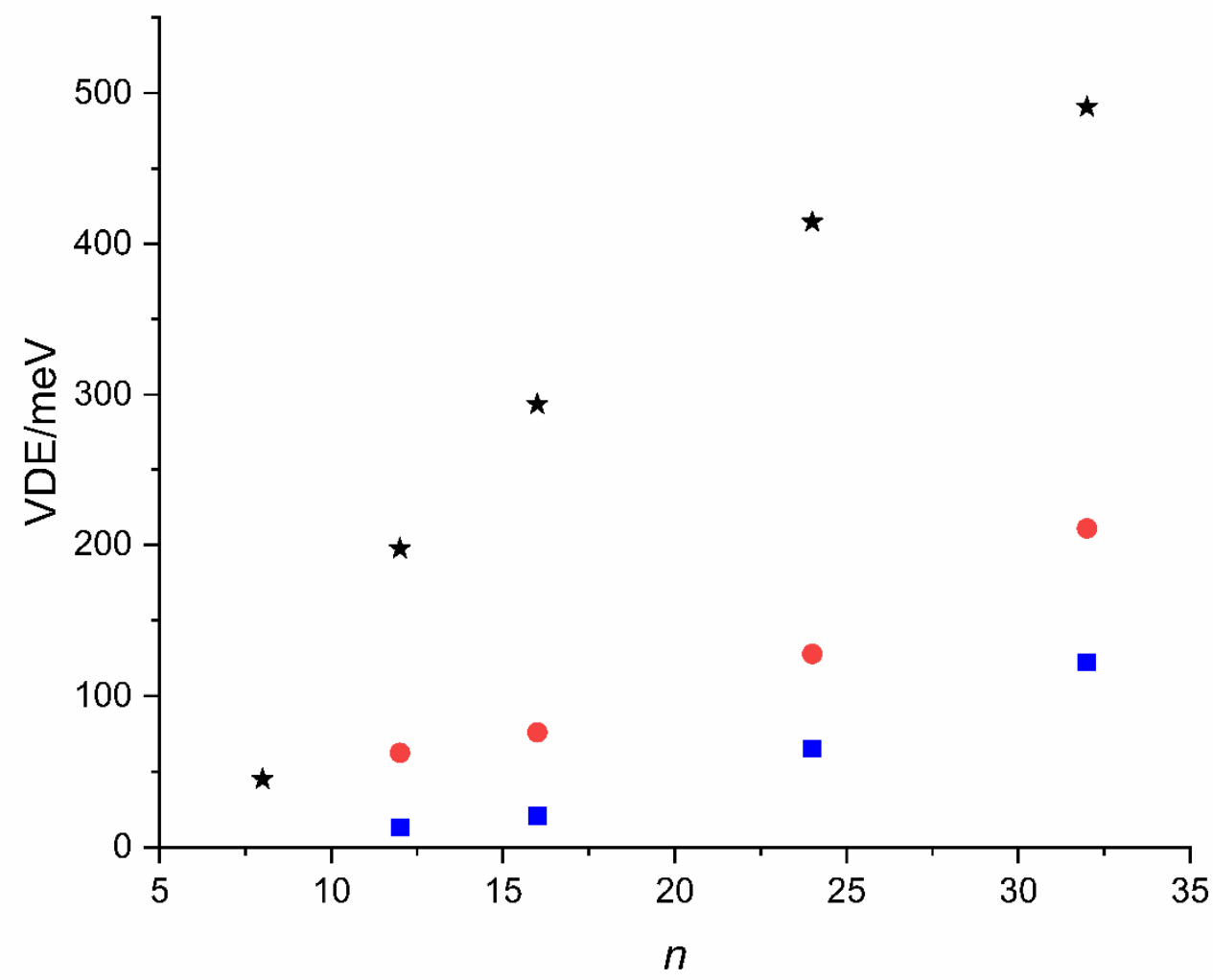




\section{References}

${ }^{1}$ W. Weyl, Annalen Der Physik Und Chemie 197, 601 (1864).

${ }^{2}$ C. A. Kraus, J. Am. Chem. Soc. 30, 1323 (1908).

${ }^{3}$ E. J. Hart, and M. Anbar, The Hydrated Electron (Wiley-Interscience: New York, NY, 1970).

${ }^{4}$ B. C. Garrett, D. A. Dixon, D. M. Camaioni, D. M. Chipman, M. A. Johnson, C. D. Jonah, G. A. Kimmel, J. H. Miller, T. N. Rescigno, P. J. Rossky, S. S. Xantheas, S. D. Colson, A. H. Laufer, D. Ray, P. F. Barbara, D. M. Bartels, K. H. Becker, K. H. Bowen, Jr., S. E. Bradforth, I. Carmichael, J. V. Coe, L. R. Corrales, J. P. Cowin, M. Dupuis, K. B. Eisenthal, J. A. Franz, M. S. Gutowski, K. D. Jordan, B. D. Kay, J. A. LaVerne, S. V. Lymar, T. E. Madey, C. W. McCurdy, D. Meisel, S. Mukamel, A. R. Nilsson, T. M. Orlando, N. G. Petrik, S. M. Pimblott, J. R. Rustad, G. K. Schenter, S. J. Singer, A. Tokmakoff, L.-S. Wang, C. Wittig, and T. S. Zwier, Chem. Rev. 105, 355 (2005).

${ }^{5}$ W. L. Jolly, Metal-Ammonia Solutions (Dowden, Hutchinson, and Ross: Stroudsburg, PA, 1972).

${ }^{6}$ M. C. R. Symons, Chem. Soc. Rev. 5, 337 (1976).

${ }^{7}$ A. Meyer, and M. van Gastel, J. Phys. Chem. A 115, 1939 (2011).

${ }^{8}$ H. W. Sarkas, S. T. Arnold, J. G. Eaton, G. H. Lee, and K. H. Bowen, J. Chem. Phys. 116, $5731(2002)$.

${ }^{9}$ C. Steinbach, and U. Buck, J. Chem. Phys. 122, 134301 (2005).

${ }^{10}$ I. A. Shkrob, J. Phys. Chem. A 110, 3967 (2006).

${ }^{11}$ T. Sommerfeld, J. Phys. Chem. A 112, 11817 (2008).

${ }^{12}$ V. V. Chaban, and O. V. Prezhdo, J. Phys. Chem. B 112, 2500 (2016).

${ }^{13}$ R. A. Ogg, J. Am. Chem. Soc. 68, 155 (1946).

${ }^{14}$ J. Jortner, J. Chem. Phys. 30, 839 (1959). 
${ }^{15}$ M. Sprik, and M. L. Klein, J. Chem. Phys. 91, 5665 (1989).

${ }^{16}$ M. Marchi, M. Sprik, and M. L. Klein, J. Phys. Chem. 94, 431 (1990).

${ }^{17}$ J. Rodriguez, M. S. Skaf, and D. Laria, J. Chem. Phys. 119, 6044 (2003).

${ }^{18}$ R. N. Barnett, U. Landman, C. L. Cleveland, N. R. Kestner, and J. Jortner, J. Chem. Phys. 88, 6670 (1988).

${ }^{19}$ M. D. Newton, J. Phys. Chem. 79, 2795 (1975).

${ }^{20}$ T. Clark, and G. Illing, J. Am. Chem. Soc. 109, 1013 (1987).

${ }^{21}$ J. A. Pople, M. Head-Gordon, and K. Raghavachari, J. Chem. Phys. 87, 5968 (1987).

${ }^{22}$ C. Møller and M. S. Plesset, Phys. Rev. 46, 0618 (1934).

${ }^{23}$ A. D. Becke, Phys. Rev. A 38, 3098 (1988).

${ }^{24}$ C. Lee, W. Yang, and R. G. Parr, Phys. Rev. B 37, 785 (1988).

${ }^{25}$ H. Iikura, T. Tsuneda, T. Yanai, and K. Hirao, J. Chem. Phys. 115, 3540 (2001).

${ }^{26}$ A. D. Becke, J. Chem. Phys. 98, 5648 (1993).

${ }^{27}$ P. J. Stephens, J. F. Devlin, C. F. Chabalowski, and M. J. Frisch, J. Phys. Chem. 98, 11623 (1994).

${ }^{28}$ A. D. Becke, J. Chem. Phys. 98, 1372 (1993).

${ }^{29}$ J. C. Rienstra-Kiracofe, G. S. Tschumper, H. F. Schaefer, S. Nandi, and G. B. Ellison, Chem. Rev. 102, 231 (2002).

${ }^{30}$ J. P. Perdew, K. Burke, and M. Ernzerhof, Phys. Rev. Lett. 77, 3865 (1996).

${ }^{31}$ C. Adamo and V. Barone, J. Chem. Phys. 110, 6158 (1999).

${ }^{32}$ M. Boero, M. Parrinello, K. Terakura, T. Ikeshoji, and C. C. Liew, Phys. Rev. Lett. 90, 226403 (2003).

${ }^{33}$ O. Marsalek, F. Uhlig, T. Frigato, B. Schmidt, and P. Jungwirth, Phys. Rev. Lett. 105, 043002 (2010). 
${ }^{34}$ O. Marsalek, T. Frigato, J. VandeVondele, S. E. Bradforth, B. Schmidt, C. Schütte, and P. Jungwirth, J. Phys. Chem. B 114, 915 (2010).

${ }^{35}$ R. N. Barnett, R. Giniger, O. Cheshnovsky, and U. Landman, J. Phys. Chem. A. 115, 7378 (2011).

${ }^{36}$ L. Mones, G. Pohl, and L. Turi, Phys. Chem. Chem. Phys. 20, 28741 (2018).

${ }^{37}$ L. Turi, J. Chem. Phys. 144, 154311 (2016).

${ }^{38}$ J. M. Herbert, and M. Head-Gordon, J. Phys. Chem. A 109, 5217 (2005).

${ }^{39}$ J. M. Herbert, and M. Head-Gordon, Phys. Chem. Chem. Phys. 8, 68 (2006).

${ }^{40}$ G. Pohl, L. Mones, and L. Turi, J. Chem. Phys. 145, 164313 (2016).

${ }^{41}$ R. M. Richard, and J. M. Herbert, J. Chem. Theory Comput. 7, 1296 (2011).

${ }^{42}$ R. Baer, E. Livshits, and U. Salzner, Annu. Rev. Phys. Chem. 61, 85 (2010).

${ }^{43}$ S. Grimme, WIREs Comput. Mol. Sci. 1, 211 (2011).

${ }^{44}$ W. J. Hehre, R. Ditchfield, and J. A. Pople, J. Chem. Phys. 56, 2257 (1972).

${ }^{45}$ M. J. Frisch, A. A. Pople, and J. S. Binkley, J. Chem. Phys. 80, 3265 (1984).

${ }^{46}$ T. H. Dunning Jr., J. Chem. Phys. 90, 1007 (1989).

${ }^{47}$ R. A. Kendall, T. H. Dunning Jr., and R. J. Harrison, J. Chem. Phys. 96, 6796 (1992).

${ }^{48}$ Gaussian 09, Revision E.01, M. J. Frisch, G. W. Trucks, H. B. Schlegel, G. E. Scuseria, M. A. Robb, J. R. Cheeseman, G. Scalmani, V. Barone, B. Mennucci, G. A. Petersson, H. Nakatsuji, M. Caricato, X. Li, H. P. Hratchian, A. F. Izmaylov, J. Bloino, G. Zheng, J. L. Sonnenberg, M. Hada, M. Ehara, K. Toyota, R. Fukuda, J. Hasegawa, M. Ishida, T. Nakajima, Y. Honda, O. Kitao, H. Nakai, T. Vreven, J. A. Montgomery, Jr., J. E. Peralta, F. Ogliaro, M. Bearpark, J. J. Heyd, E. Brothers, K. N. Kudin, V. N. Staroverov, R. Kobayashi, J. Normand, K. Raghavachari, A. Rendell, J. C. Burant, S. S. Iyengar, J. Tomasi, M. Cossi, N. Rega, J. M. Millam, M. Klene, J. E. Knox, J. B. Cross, V. Bakken, C. Adamo, J. Jaramillo, R. Gomperts, R. E. Stratmann, O. Yazyev, A. J. Austin, R. Cammi, C. Pomelli, J. W. Ochterski, R. L. Martin, 
K. Morokuma, V. G. Zakrzewski, G. A. Voth, P. Salvador, J. J. Dannenberg, S. Dapprich, A. D. Daniels, Ö. Farkas, J. B. Foresman, J. V. Ortiz, J. Cioslowski, and D. J. Fox, Gaussian, Inc., Wallingford CT, 2009.

${ }^{49}$ M. J. S. Dewar, E. G. Zoebisch, E. F. Healy, and J. J. P. Stewart, J. Am. Chem. Soc. 107, 3902 (1985).

50 J. VandeVondele, M. Krack, F. Mohamed, M. Parrinello, T. Chassaing, and J. Hutter, Comput. Phys. Commun. 167, 103 (2005).

${ }^{51}$ S. Goedecker, M. Teter, and J. Hutter, Phys. Rev. B 54, 1703 (1996).

${ }^{52}$ C. Hartwigsen, S. Goedecker, and J. Hutter, Phys. Rev. B 58, 3641 (1998).

${ }^{53}$ J. VandeVondele, and J. Hutter, J. Chem. Phys. 127, 114105 (2007).

${ }^{54}$ M. Guidon, J. Hutter, and J. J. VandeVondele, J. Chem. Theory Comput. 6, 2348 (2010).

${ }^{55}$ G. J. Martyna, and M. E. Tuckerman, J. Chem. Phys. 110, 2810 (1999).

${ }^{56}$ G. Bussi, D. Donadio, and M. Parrinello, J. Chem. Phys. 126, 014101 (2007).

${ }^{57}$ L. Turi, Á. Madarász, and P. J. Rossky, J. Chem. Phys. 125, 014308 (2006).

${ }^{58}$ L. Mones, P. J. Rossky, and L. Turi, J. Chem. Phys. 133, 144510 (2010).

${ }^{59}$ H. M. Lee, S. Lee, and K. S. Kim, J. Chem. Phys. 119, 187 (2003).

${ }^{60}$ A. Lietard, and J. R. R. Verlet, J. Phys. Chem. Lett. 10, 1180 (2019). 